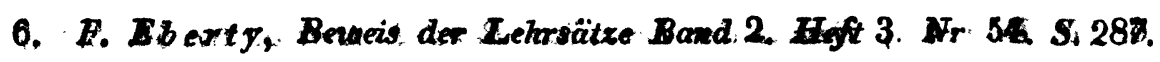

\title{
6.
}

\section{Beweis der Lehrsätze Band 2. Heft 3. Nr. 54. 8. 287.}

(Von Herrn Felix Eberty zu Berlin.)

,1. Fället man aus einem willkürlichen Puncte $D$ (Taf. I. Fig. \%.) in der Ebene eines Dreiecks $A B C$, auf die Seiten des leteteren Iuothe, $D a$, $D b$ und $D c$, nimmt in diesen Lothen drei beliebige Puncte $a, b, c$ als Ecken eines andern Dreiecks $a b c$ an, und fället auf dessen Seiten ans den Ecken des gegebenen Dreieeks, in gehöriger Ordnung genommen, Lothe, $A d, B d, C d$, so treffen dieso einander allemal in irgend einem Puncte $d$," und ferner:

„H. Nimmt man ähnlicher Weise ein drittes Dreieck an, $a_{1} b_{2} c_{1}$, dessen Ecken in den nemlichen drei ersten Lothen liegen, so wird demselben auf gleiche Weise ein Punct $d_{1}$ entsprechen ( $\mathrm{I}_{0}$ ), und alsdann liegen die drei Durchschnittspuncte der drei Paar entsprechenden Seiten des zweiten Dreiecks mit denen des dritten, das heifst die Durchschnittspuncte $\alpha, \beta$ und $\gamma$ der Seitenpaare $b c$ und $b_{1} c_{1}, c a$ und $c_{1} a_{1}, a b$, and $a_{1} b_{1}$ ellemal in Einer Geraden, $\alpha \beta \gamma$, , und

„III. Diese Gerade ist allemal zu derjenigen Geraden $d d_{1}$, welch durch die beiden genannten Puncte $d$ und $d_{1}$ geht, senkrecht."

An diese Lehrsätze schliefst sich noch folgender an:

IV. Nimmt man in jeder von dreien sich in Einem Puncte schneidenden Geraden (Fig. 8.) zwei beliebige Puncte an, und bezeichnet die in jeder derselben dem Durchschnittspuncte zunächst liegenden mit $a, b$ und $c$, die andern drei mit $a_{1}, b_{1}$ und $c_{1}$, so liegen die Durchschnittspuncte der Verbindungslinien $a b, b c$ und $a c$, mit den Verbindungelinien $a_{2} b_{2}, b_{2} c_{2}$ und $\alpha_{1} c_{1}, \mathrm{~d}$. h. die Puncte $\alpha, \beta$ und $\underline{\gamma}$ allemal in einer Geraden.

B ow e is von I. Man beschreibe aus den Punctea $A, B$ und $C$ (Fig. 7.) drei Kreise, deren zwei immer die auf ihrer Axo senkrecht stehenden Geraden $D a, D b$ und $D c$ za Linien der gleichen Potenzen haben, welches auf folgende Art möglich ist. Man beschreibe um $c$ mit einem beliebigen Radius $c x$ einen Kreis, lege sodann von $A$ aus an den14 * 
108 6. E. Bberty, Beweis der Lehrsätze Band 2. Heft 3. Nr. 54. S. 287.

selben eine Tangente $A a$, und beschreibe mit $A x$ um $A$ einen Kreis, desgleichen mit $B y$ (einer von $B$ aus an den Kreis um $c$ gezogenen Tangente) um $B$, sodann mit $a z$ (einer Tangente an den Kreis um $B$ ) einen Kreis um $a$, und zuletzt mit $C t$ (einer Tangente an den Kreís um $a$ ) einen Kreis um $C$, so sind die drei nun um $A, B$ und $C$ beschriebenen Kreise von der verlangten Art. $Z u$ denen um $A$ und $B$ nämlich ist $D c$, zu denen um $B$ und $C, D a$, und also zu denen um $A$ und $C, D b$ die Linie der gleichen Potenzen. (S. die Abhandlungen des Herrn Steiner in diesem Journal, Band 2.) Also kann man auch aus $b$ mit $b m$ einen Kreis beschreiben, welcher die um $A$ und $C$ rechtwinklig schneidet (wie es schon vorher bei den Kreisen um $c$ und $a$, in Bezug auf die um $B$ und $A$, und die um $C$ und $B$ der Fall war). Der Kreis um $C$ also wird, wie man. sahe, von dem um $a$ sowohl, als von dem um $b$ rechtwinklig geschnitten, folglich liegt $C$ in der Linie der gleichen Potenzen der Kreise um $a$ und $b$, und zwar stellt demnach das von $c$ auf $a b$ gefällte Perpendikel (s. jene Abhandlungen) diese Linie der gleichen Potenzen dar. Auf dieselbe Weise folgt, dafs auch das von $A$ auf $c b$ gefällte Perpendikel zu den Kreisen um $c$ und $b$, und das von $B$ aus auf $a c$ gefällte, für die Kreise um $a$ und $b$, Linien der gleichen Potenzen sind. Es sind also die drei Lothe $A d, B d$ und $C d$ Linien der gleichen Potenzen zu je zweien von den drei Kreisen $a, b$ und $c$; sie scheiden sich demnach (s. jene Abhandlungen) alle drei in Einem Puncte; was zu beweisen war.

Beweis von II. und III. $d_{1}$ also ist der Punct, welcher im Dreieck $a_{1} b_{1} c_{1}$ dem Puncte $d$ im Dreieck $a b c$ entspricht. Die Seiten $a b$ und $a_{2} b_{1}$ schneiden sich in $\alpha_{1} b c$ und $b_{1} c_{1}$ in $\gamma$ und $a c$ und $a_{1} c_{3}$ in $\beta$. Es ist zu beweisen, dafs die Puncte $\alpha, \beta$ und $\gamma$ in einer geraden Linie liegen, welche mit der durch $d$ und $d_{1}$ gezogenen einen rechten Winkel bildet. Man lege um $d$ einen Kreis, welcher die um $a, b$ und $c$ rechtwinklig durchschneidet (welches angeht, weil in $d$ die Linien der gleichen Potenzen für jene Kreise (um $a, b$ und $c$ ) sich treffen), eben so aus $d_{1}$ einen Kreis, welcher die um $a_{1}, b_{x}$ und $c_{x}$ rechtwinklig durchschneidet (welches aus ganz ähnlichem Grunde möglich ist). $a b$ ist die Linio der gleichen Potenzen für die Kreise um $C$ und $d$ (denn beide werden von denen um $a$ und $b$ rechtwinklig geschnitten); aus demselben Grunde ist auch $a_{1} b_{1}$ die Linie der gleichen Potenzén für die Kreise um $C$ und um $d_{2}$. Jeder Punct in 
6. F. Eberty, Belveis der Lehrsätze Band 2. Heft 3. Nr. 54. S. 287.109

der Linie $a b$ hat also für die Kreise um $C$ und $d$, jeder Punct in der Linie $a_{1} b_{2}$ für die Kreise um $C$ und $d_{2}$ gleiche Potenzen; folglich hat ihr Durchschnittspunct (a) auch für die Kreise um $d$ und um $d_{3}$ gleiche Potenzen; es liegt also $a$ in der Linie der gleichen Potenzen für die Kreise um $d$ und um $d_{3}$. Dasselbe ist auf dieselbe Weise von $\gamma$ und $\beta$ zu beweisen. Es liegt also $\alpha$ sowohl als $\beta$ und $\gamma$ in der Linie der gleichen Potenzen für die Kreise um $d$ und um $d_{3}$, alle drei also liegen in einer einzigen geraden Linie, und zwar (s. jene Abhandlungen) in einer geraden Linie, welche auf der durch $d$ und $d_{2}$ gezogenen senkrecht steht; denn $d d_{3}$ ist die Axe der beiden um diese Puncte beschriebenen Kreise.

Beweis von IV. Mit wechselnder Grölse der Kreise um $A, B$ und $C$, welche ursprünglich ganz beliebig angenommen sind, wechseln auch die Gröfsen der Winkel, unter welchen die drei Geraden $a D, b D$ $c D$ einander schneiden, und zwar bei allmäligem Wachsen oder Abnehmen jener Kreise, allmälig wachsend oder abnehmend. Da demnach diese Winkel jede mögliche Gröfse haben können, so folgt unmittelbar, dafs die in den drei geraden Linien $D a, D b$ und $D c$ beliebig angenommenen 6 Puncte, $a, b, c$ und $a_{1}, b_{1}, c_{1}$, auch in jeden andern drei sich in einem Puncte unter beliebigen Winkeln schnoidenden Linien angenommen werden können, so dafs die drei Puncte $\alpha, \beta$ und $\gamma$ immer in Einer Geraden liegen; was $z u$ beweisen war.

Auch die Umkehrung aller dieser Sätze läfst sich darthun. 